\title{
Kiddie-SADS Reveals High Rates of DSM-IV Disorders in Children and Adolescents with Autism Spectrum Disorders
}

\author{
Elen Gjevik $\cdot$ Sigmund Eldevik $\cdot$ \\ Torill Fjæran-Granum • Eili Sponheim
}

Published online: 8 September 2010

(c) The Author(s) 2010. This article is published with open access at Springerlink.com

\begin{abstract}
Prevalence of current comorbid DSM-IV disorders was assessed in a special school population of children and adolescents with ASD $(N=71$, age 6.0-17.9 years), representing all cognitive levels and main ASD subgroups. Symptoms were assessed through parent interview and association to child characteristics was explored. Seventy-two percent was diagnosed with at least one comorbid disorder. Anxiety disorders (41\%) and attention deficit/hyperactivity disorder $(31 \%)$ were most prevalent. Obsessive-compulsive disorder was more common in older children, and oppositional defiant disorder/ conduct disorder more prevalent in pervasive developmental disorder, not otherwise specified. Our results show high rates of comorbid DSM-IV disorders and underscore the importance of such evaluation in children ASD. However, diagnostic challenges are present and future research on the diagnostic validity of comorbid psychiatric disorders is needed.
\end{abstract}

\author{
E. Gjevik $(\bowtie) \cdot$ E. Sponheim \\ Division of Mental Health and Addiction, Oslo University \\ Hospital, P.O. Box 26, 0319 Vinderen, Oslo, Norway \\ e-mail: elen.gjevik@medisin.uio.no \\ E. Gjevik \\ Institute of Clinical Medicine, University of Oslo, Oslo, Norway \\ S. Eldevik · T. Fjæran-Granum \\ Nordvoll School and Centre of Autism, Oslo, Norway \\ Present Address: \\ S. Eldevik \\ Faculty of Behavioral Science, Akershus University College, \\ Oslo, Norway \\ Present Address: \\ T. Fjæran-Granum \\ SPISS, Oslo, Norway
}

Keywords ASD · Children-adolescents · Comorbid psychiatric disorders

\section{Introduction}

Autism spectrum disorders (ASD) are clinically heterogeneous disorders with a wide range in severity of the core features and seen in individuals of all cognitive level (American Psychiatric Association 2000; Geschwind 2009). In clinical practice it has long been recognised that children and adolescents with ASD often have comorbid psychopathology contributing further to the broad variability in the ASD clinical presentation. Comorbid symptoms and disorders represent an added handicap for the affected child, require considerable attention and become a focus for intervention and medical treatment (Gadow et al. 2008; Holtmann et al. 2007; Weisbrot et al. 2005).

A growing number of systematic studies confirm clinical observations and report comorbid psychiatric symptoms and disorders to be common in children with ASD.

High symptom scores are reported for attention deficit/ hyperactivity (Yoshida and Uchiyama 2004), anxiety (Sukhodolsky et al. 2008; Weisbrot et al. 2005), depression and aggressive behaviour (Brereton et al. 2006). Studies assessing psychiatric disorders report attention deficit/ hyperactivity disorder (ADHD) and anxiety to be particularly frequent (Lee and Ousley 2006; Simonoff et al. 2008; Leyfer et al. 2006; Muris et al. 1998), while findings on depression and oppositional defiant disorder/conduct disorder $(\mathrm{ODD} / \mathrm{CD})$ are more inconsistent (Simonoff et al. 2008; Ghaziuddin et al. 2002; Leyfer et al. 2006; de Bruin et al. 2007). To date knowledge on psychiatric comorbidity in children with ASD derives mainly from questionnaire 
studies providing symptom scores, while standardized interview studies yielding DSM-IV diagnoses are limited (Leyfer et al. 2006; Simonoff et al. 2008; Bradley and Bolton 2006). Moreover, most previous studies have included clinically referred samples and focused on children with high functioning autism and Asperger's syndrome. There are few studies including more severe samples. Associations between child characteristics and comorbid psychiatric symptoms are studied in questionnaire studies (Sukhodolsky et al. 2008; Weisbrot et al. 2005; Brereton et al. 2006; Sinzig et al. 2009), while knowledge of child characteristics in relation to comorbid psychiatric disorders is scarce (Simonoff et al. 2008).

Diagnosing comorbid psychiatric disorders in the presence of ASD is a matter of controversy. The hierarchical and categorical structure of the DSM-IV implies that psychiatric symptoms should not be diagnosed in clinical practice if better accounted for by a more severe disorder (American Psychiatric Association 2000). As for ASD this may lead to a tendency to attribute all presenting symptoms to the ASD. There are some diagnoses that DSM-IV mentions explicitly when it comes to being diagnosed in the presence of ASD. ADHD should not be diagnosed if exclusively occurring during the course of ASD, and excluded in autistic disorder. Moreover, generalized anxiety disorder is precluded by DSM-IV if ASD is present. However, these guidelines are based on consensus over diagnostic procedure, and not grounded on research showing that all presenting symptoms necessarily are part of ASD. Hence, it is increasingly argued that comorbid psychiatric disorders should be assessed and diagnosed when present (Simonoff et al. 2008). Assessing comorbid psychiatric disorders in children and adolescents with ASD presents diagnostic challenges. Standardized diagnostic interviews used in the general child population are not validated in children with ASD, and few specialized instruments are developed (Leyfer et al. 2006; Bradley and Bolton 2006). Further, due to impairment in language and cognition children with ASD often have difficulty communicating their thoughts and feelings, complicating the evaluation of psychiatric symptoms. Lastly, symptoms of comorbid psychiatric disorders and core features of ASD may be overlapping and difficult to distinguish. Notably, obsessive-compulsive disorder (OCD) may be difficult to distinguish from repetitive and ritualistic behaviour, tic disorders from stereotyped and repetitive movements, social phobia from impairment of social interaction and psychotic symptoms from stereotyped and repetitive thinking (Zandt et al. 2007; Dossetor 2007).

The aim of this study was to assess the prevalence of current comorbid DSM-IV disorders in a special school population of children and adolescents with ASD representing the broad range of intellectual levels and main ASD subgroups. DSM-IV exclusionary criteria for ASD were not applied. All psychiatric symptoms were identified and comorbid disorders recorded when diagnostic criteria were met. We also explored associations between comorbid psychiatric disorders and age, gender, cognitive level, language ability and ASD subgroups.

\section{Methods}

Participants

The study participants were recruited from a population of 94 children and adolescents registered at the only special school for students with ASD in Oslo, Norway. Children registered at the special school were either students at the special school, or students at regular schools receiving, or having applied for counselling from the special school. Parents and children received written information about the study, while participation was based on written consent from the parents. A total of 71 children and adolescents were included (response rate $76 \%$ ).

Out of the children not included $(N=23)$, two were excluded due to parental insufficient language skills (Norwegian and English), and one because she did not fulfill the diagnostic criteria for ASD. Fourteen refused to participate and 6 did not respond to the invitation.

\section{Measures}

The schedule for Affective Disorders and Schizophrenia for School-Age Children-Present and Lifetime version (Kiddie-SADS-PL), a semi-structured interview based on DSM-IV (Kaufman et al. 1997), was used to assess comorbid psychiatric disorders. Current comorbid psychiatric disorders were recorded. The interview was performed with one or both parents. Kiddie-SADS consists of a screening interview and 6 diagnostic supplements. The screening interview was conducted for all participants, and the supplements when indicated from the results of the screening interview. Two clinicians (ES and EG), both experienced with children with ASD, undertook the interviews.

Inter-rater agreement was assessed on 18 interviews: the first five consecutive cases and randomly in the rest of the sample. Both clinicians were present during these interviews, but were blind to each other's ratings. Interviewing alternated between the clinicians. Twenty-two diagnoses were established in these 18 interviews. There was full agreement on 19 diagnoses (86\%). Disagreement was on tic disorder in two children, and ADHD in one child. These 
latter cases were discussed between the two clinicians and diagnoses set in consensus. Finally, all 71 interviews were reassessed jointly by the two clinicians and diagnoses established by consensus best estimate.

Autism Diagnostic Interview-Revised (ADI-R) is a semi-structured parent interview classifying children as meeting or not meeting the criteria for autistic disorder (Lord et al. 1997). All study participants were previously diagnosed with ASD. Nevertheless, all were reassessed using ADI-R by one of the authors (SE), who is certified as an interviewer for research purpose. Based on all available information, including scores from ADI-R, cognitive level and age at debut of ASD symptoms, the participants were diagnosed according to DSM-IV criteria for PDD, and corresponded to the main ASD subgroups; autistic disorder, Asperger's disorder and PDD NOS. Associations between each of the three ASD subgroups and comorbid psychiatric disorders were tested.

Vineland Adaptive Behavior Scales is a semi-structured parent interview that measures competence in communication, daily living skills and socialization (Sparrow et al. 1984). Scores on the communication sub domain were used to explore associations between functional communication skills and comorbid psychiatric disorders $(N=69)$.

Leiter international performance Scale-Revised (Leiter-R) is a non-verbal measure for learning and problem solving abilities, and consists of two batteries, an Attention/Memory Battery and Visualisation/Reasoning Battery (Roid and Miller 1997). Only the latter was administrated in this study. The score from Leiter-R was used to explore associations between non-verbal intellectual level and comorbid psychiatric disorders $(N=69)$.

Receptive One-Word Picture Vocabulary Test (ROWPVT-R) measures receptive single-word vocabulary (Gardner 2000), and was used in this study because it can assess those with little or no language. ROWPVT-R scores were used to explore associations between receptive language skills and comorbid psychiatric disorders $(N=69)$.

\section{Statistical Methods}

Non-verbal IQ, age, receptive and functional language skills were treated as continuous variables. Two-sample $T$-tests were performed to explore differences between groups (children with comorbid disorder vs. children with no comorbid disorder) on continuous variables. Exact chisquare tests were conducted to compare groups on categorical variables (gender and ASD subgroup). All $p$ values were presented two-tailed, and $p$ values less than 0.05 are reported as significant. SPSS version 15.0 and 16.0 were used for the statistical analyses.

\section{Results}

\section{Sample Characteristics}

The sample included children and adolescents, between 6.0 and 17.9 years of age, of all cognitive levels and main ASD subgroups. Male: female ratio was 4.5:1.0. Sample characteristics are presented in more detail in Table 1.

Prevalence of Comorbid Psychiatric Disorders

Fifty-one children ( $72 \%$ of the sample) were diagnosed with at least one comorbid psychiatric disorder. The most prevalent diagnostic groups were anxiety disorders, diagnosed in 30 children (42\%), and ADHD diagnosed in 22 children $(31 \%)$. ADHD, predominantly inattentive type, was the most prevalent ADHD subgroup (21\% of all children and $68 \%$ of those with ADHD). Specific phobia was the most prevalent anxiety disorder, diagnosed in 22 children $(31 \%)$. Phobia of loud noises was a frequent expression of phobia, recorded in 10 children. Phobia of dogs and specific situations was also common. Five children $(7 \%)$ met criteria for social phobia and 7 (10\%) for

Table 1 Child characteristics

\begin{tabular}{|c|c|c|}
\hline & $N$ & $\%$ \\
\hline \multicolumn{3}{|l|}{ Gender } \\
\hline Male & 58 & 82 \\
\hline Female & 13 & 18 \\
\hline \multicolumn{3}{|c|}{ ASD subgroup $(N=71)$} \\
\hline Autistic disorder & 47 & 66 \\
\hline Asperger's disorder & 12 & 17 \\
\hline PDD NOS & 12 & 17 \\
\hline \multicolumn{3}{|l|}{ Age (years; $N=71$ ) } \\
\hline$<11$ years & 34 & 48 \\
\hline$\geq 11$ years & 37 & 52 \\
\hline \multicolumn{3}{|c|}{ Mean $(\mathrm{SD}) ;$ range $=11.8(3.3) ; 6.2-17.9$} \\
\hline \multicolumn{3}{|c|}{ Nonverbal IQ, Leiter-R $(N=69)$} \\
\hline$\leq 34$ & 17 & 25 \\
\hline $35-69$ & 22 & 32 \\
\hline$\geq 70$ & 30 & 43 \\
\hline \multicolumn{3}{|c|}{ Mean $(\mathrm{SD}) ;$ range $=65.2(29.6) ; 30-129$} \\
\hline \multicolumn{3}{|c|}{ Functional language, Vineland $(N=69)$} \\
\hline$\leq 34$ & 30 & 43 \\
\hline $35-69$ & 26 & 38 \\
\hline$\geq 70$ & 13 & 19 \\
\hline \multicolumn{3}{|c|}{ Receptive language, ROWPVT-R $(N=69)$} \\
\hline$\leq 69$ & 41 & 59 \\
\hline$\geq 70$ & 28 & 41 \\
\hline
\end{tabular}


Table 2 Comorbid DSM-IV disorders in 71 children with ASD

\begin{tabular}{lrr}
\hline & $N$ & $\%$ \\
\hline Any psychiatric disorder & 51 & 72 \\
Any anxiety disorder & 30 & 42 \\
Specific phobia & 22 & 31 \\
Social phobia & 5 & 7 \\
Obsessive-compulsive disorder & 7 & 10 \\
Separation anxiety disorder & 0 & \\
Generalized anxiety disorder & 0 & \\
Any ADHD & 22 & 31 \\
ADHD, inattentive type & 15 & 6 \\
ADHD, combined type & 3 & 10 \\
ADHD, hyperactive type & 4 & 7 \\
Any mood disorder & 7 & \\
Depressive disorder, NOS & 5 & \\
Major depressive disorder & 1 & \\
Dysthymic disorder & 1 & \\
Manic episode/bipolar disorder & 0 & \\
Any tic disorder & 8 & \\
Oppositional defiant disorder & 3 & \\
Conduct disorder & 2 & \\
Any psychotic disorder & 1 & \\
Anorexia nervosa/bulimia & 0 & \\
\hline & &
\end{tabular}

Twenty-one children had more than one comorbid DSM-IV disorder Four children had three comorbid disorders and one child had four

OCD. Children with OCD had symptoms such as worries about contamination, checking and hand washing. Any depressive disorder was diagnosed in 7 children (10\%). Depressive disorder NOS was the most prevalent subtype of depression, diagnosed in 5 children (7\%). Any tic disorder was diagnosed in 8 children $(11 \%)$. Five children (7\%) received an ODD/CD diagnosis and one child was diagnosed with a psychotic disorder. Prevalence of comorbid psychiatric disorders is presented in Table 2.

\section{Multiple Psychiatric Disorders}

Twenty-one children (41\% of those with any comorbid disorder, and $30 \%$ of the sample) were diagnosed with more than one disorder. Four children had three comorbid disorders, and one child had four. Ten of the 22 children with ADHD had more than one comorbid disorder, most frequently an anxiety disorder. Overlap between ODD/CD and ADHD was only diagnosed in one child. Four children had both a depressive disorder and an anxiety disorder. Out of the 30 children with any anxiety disorder, 4 met criteria for more than one anxiety disorder. Overlap between main diagnostic groups is illustrated in Fig. 1.

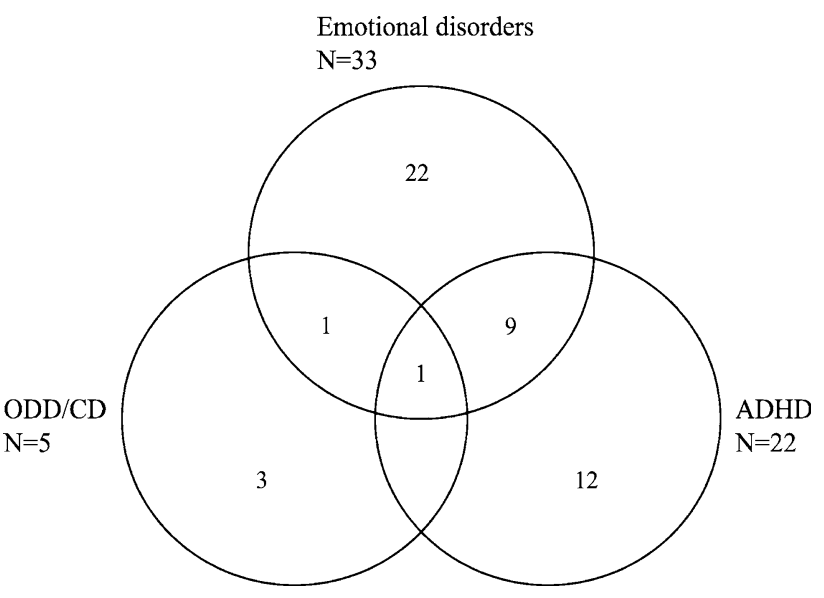

Fig. 1 Overlap between major diagnostic groups in 48 of 51 children diagnosed with at least one comorbid psychiatric disorder. Emotional disorders denote any depressive disorder $(n=7)$ and any anxiety disorder $(n=30)$. Four children had both an anxiety disorder and an depressive disorder. Out of the 10 children who were diagnosed with both ADHD and an emotional disorder 9 had an anxiety disorder. Two children with tic disorder only and one child with psychotic disorder only are not included

\section{Associations to Child Characteristics}

Associations between child characteristics and comorbid psychiatric disorders were tested for any psychiatric disorder, the main diagnostic groups (anxiety disorders, ADHD, depressive disorders, tic disorders and ODD/CD) and for the subtypes of psychiatric disorders that included five or more children with the diagnoses (OCD, social phobia, specific phobia, ADHD inattentive type and depressive disorder, NOS). Children with OCD were found to be significantly older (Mean $=15.5, \mathrm{SD}=2.5$ ) than those without $\mathrm{OCD}($ Mean $=11.4, \mathrm{SD}=3.2 ; p=0.001)$. ODD/CD was more prevalent in children with PDD NOS than in those without this disorder (Asperger's syndrome or autistic disorder; $p=0.03$ ). No other associations were found between comorbid psychiatric disorder and age, gender, intellectual level, receptive language and ASD subgroups.

\section{Discussion}

In this study we used Kiddie-SADS, a semi-structured interview based on DSM-IV criteria, to assess the prevalence of current comorbid psychiatric disorders in a special school population of children and adolescents with ASD. Seventytwo percent of the sample was diagnosed with at least one comorbid psychiatric disorder. The prevalence of comorbid disorders in our study exceeds the rate of psychiatric disorders both in the general child and adolescent population (7.0-13.3\%) (Heiervang et al. 2007; Costello et al. 2003), 
and in children with intellectual disabilities (38.6\%) (Dekker and Koot 2003). There are few studies that have used similar methodology as us to assess comorbid psychiatric disorders in ASD child population. Leyfer et al. (2006) and Simonoff et al. (2008) used standardized interviews and diagnosed according to DSM-IV criteria. Leyfer et al. developed a specialized instrument, Autism Comorbidity Interview (ACI), a modified version of Kiddie-SADS, and reported lifetime disorder. The main difference between ACI and Kiddie-SADS, as used in this study, was the diagnostic criteria for OCD, which in ACI were widened to include sign and symptoms observed by others. Simonoff et al. used Child and Adolescent Psychiatric Assessment (CAPA) in a population representative sample, but their sample had a more narrow age span than ours. The rate of at least one comorbid psychiatric disorder in our study corresponded to that in both Simonoff et al. and Leyfer et al. (72 and 70.8\%, respectively). Further, ADHD and anxiety disorders were among the most prevalent diagnostic groups in all three studies. However, there were noteworthy differences in the prevalence of some psychiatric disorders, as discussed below.

\section{Anxiety Disorders}

Our $42 \%$ rate of any anxiety disorder agrees with several previous reports (Simonoff et al. 2008; Leyfer et al. 2006). Specific phobia was the most prevalent anxiety disorder (31\%), as also reported by others (Muris et al. 1998; de Bruin et al. 2007; Leyfer et al. 2006). Simonoff et al. found lower rates of specific phobia (8.5\%), but reported agoraphobia and panic disorder at similar rates as specific phobia. In contrast, agoraphobia and panic disorder were diagnosed neither in the Leyfer et al. sample nor in our sample. However, all studies agree on the finding that focused symptoms of anxiety are prevalent in children and adolescents with ASD. We found, similar to Leyfer et al., phobia of loud noises to be the most frequent expression of specific phobia.

Social phobia was diagnosed in $7 \%$ of our sample, a finding in line with Leyfer et al. (7.5\%). Simonoff et al. reported higher rates (29.2\%), as did Muris et al. (20.5\%) (Muris et al. 1998). Muris' study included a clinical sample and the majority had PDD NOS. Rate differences could reflect sample dissimilarities as well as different ways of interpreting and defining symptoms of anxiety. A key question may be whether avoidance in social interaction is to be interpreted as a symptom of anxiety or a feature of ASD. Our interpretation of the DSM-IV diagnostic criteria is that social phobia requires observable and expressed symptoms of anxiety, and that avoidance in social interaction alone is insufficient for a diagnosis.
Ten percent of our sample was diagnosed with OCD. Simonoff et al. and Muris et al. found comparable rates (8.5 and $11.4 \%$, respectively), while Leyfer et al., used modified criteria for OCD and reported considerably higher prevalence $(37 \%)$. We agree with authors emphasizing the difficulty of distinguishing OCD symptoms from repetitive behaviour in ASD (Zandt et al. 2007). DSM-IV indicates a qualitative distinction: repetitive behaviour being a source of pleasure in ASD, while associated with anxiety and distress in OCD. However, anxiety and distress related to repetitive and stereotyped behaviour may easily be overlooked in children and adolescents with ASD.

No children in our sample were diagnosed with separation anxiety disorder. Parents often described resistance to change in routine and new settings. However, symptoms of anxiety related to being separated from their parents or being away from home were not reported. Our findings are in line with Simonoff et al. $(0.5 \%)$, while Leyfer et al. and Muri et al. reported higher rates (11.9 and 27.3\%, respectively).

Generalized anxiety disorder, GAD, was not diagnosed in our sample. The subjective symptoms underlying a GAD diagnosis may have been missed due to our indirect diagnostic procedure, relying on parent information only. Nevertheless, parents did not report objective symptoms of GAD such as being generally tense or distressed. Leyfer et al. reported low rates of GAD (2.4\%). Simonoff et al. found higher rates (13.4\%), as did Muri et al. of overanxious disorder (22.7\%). The absence of generalized expressions of anxiety, GAD and separation anxiety disorders could also reflect the predominance of children with autistic disorder in our sample, and relatively many with low age and cognitive level.

\section{Attention Deficit/Hyperactivity Disorder}

High rate of ADHD is a consistent finding in previous studies. Our $31 \%$ rate is in line with Leyfer et al. and Simonoff et al., while rates as high as $53-78 \%$ has been reported in clinically referred samples (Sinzig et al. 2009; Lee and Ousley 2006). Few studies have looked at the distribution of ADHD subtypes in ASD child and adolescent populations. We found, in line with Leyfer et al. and Sinzig et al., ADHD predominantly inattentive type to be the most prevalent ADHD subtype. It could be argued that ADHD is particularly problematic to diagnose in the presence ASD, as reflected by the DSM-IV exclusionary criteria. Impairment of attention is persistently reported in individuals with ASD (Allen and Courchesne 2001), and fulfilling the criteria for ADHD, and especially ADD, could reflect these deficits. Not surprising, there is an ongoing debate whether ADHD is best conceptualized as a distinct comorbid disorder or a part of the ASD diathesis (Sinzig 
et al. 2009). Moreover, the sensitivity and specificity of a standardized interview for diagnosing ADHD in the presence of ASD needs further investigation.

The little overlap between ADHD and ODD/CD in our sample is noteworthy and inconsistent with findings in ADHD child populations (Biederman et al. 2008). However, disruptive disorders are more commonly comorbid with ADHD hyperactive subtypes than to ADD (Diamond 2005), which was the predominant ADHD subtype in our sample.

\section{Mood Disorders}

Ten percent of our sample was diagnosed with any depressive disorder. Other studies have reported comparable findings (Leyfer et al. 2006). Simonoff et al. found lower rates $(1 \%)$, however, reported that $10.9 \%$ of the sample had significant symptoms of depression, but fell short of fulfilling criteria for a diagnosis. Depressive disorder NOS was the most prevalent subtype of depression in our sample. Children with depressive disorder NOS showed the objective and observable symptoms of depression: loss of interest, irritability, anger and apathy. However, not enough subjective symptoms were recorded to diagnose a more specific depressive disorder. The predominance of depressive disorder NOS could reflect that assessment was based on parent information as well as the fact that children with ASD often have difficulties expressing their thoughts and feelings. Hence, special attention should be given to objective symptoms when diagnosing depression in ASD.

Manic episode or bipolar disorder was not diagnosed in our sample. Children were often reported to have episodes of arousal and euphoria. However, this behaviour was related to specific situations and did not persist over time, and hence did not fulfil the criteria for a disorder. Leyfer et al. reported low rate $(<2 \%)$ of bipolar disorder, as did de Bruin et al. (3.2\%) in a referred sample including children and adolescents with PDD NOS (de Bruin et al. 2007). In contrast, Wozniak et al. reported $20 \%$ prevalence in a clinically referred sample (Wozniak et al. 1997). The deviance between studies could reflect sample differences, as well as dissimilarity in defining manic symptoms.

\section{Tic Disorder}

Eleven percent in our sample was diagnosed with a tic disorder. In two children serious self-injurious behavior had the quality of tics and fulfilled criteria for the disorder. Previous studies have reported rates of a tic disorder ranging from 13.8 to $22 \%$ (Canitano and Vivanti 2007; Simonoff et al. 2008). Difficulties in distinguishing tic disorder from other movement abnormalities could underlie inconsistency in reported rates.

\section{Oppositional Defiant Disorder/Conduct Disorder}

Three children met criteria for ODD and 2 for CD (ODD/ CD prevalence $7 \%$ ). Our findings are in line with Leyfer et al. $(7 \%)$, whereas Simonoff et al. reported high rates of ODD $(28.1 \%)$, but low rates of CD (3.2\%). Aggression and acting out problems are reported to be extremely common in children with ASD (Brereton et al. 2006). The fairly low prevalence of ODD/CD in our sample, and some other studies, could indicate that the ODD/CD diagnosis does not capture the essential aspects of behavioral problems in many children with ASD. Features of ODD/CD, such as lying and blaming others, might demand a fairly mature cognitive level and ability of abstract thinking, and could be difficult to comprehend for children with ASD. We found ODD/CD to be more prevalent in children with PDD NOS than in those without this disorder. The PDD NOS children in our sample showed less severe ASD symptoms and had a relatively high cognitive level. Hence, our finding supports the notion that the expression of behavioral problems, and whether the diagnostic criteria for ODD/CD are reached, may depend upon the child's developmental level. Noteworthy, de Bruin et al. reported a $37.2 \%$ ODD prevalence rate and $9.6 \% \mathrm{CD}$ in their referred PDD NOS sample.

\section{Other Disorders}

One adolescent with PDD NOS was diagnosed with a psychotic disorder and showed a substantial loss in everyday functioning following the development of psychotic symptoms. Few studies have assessed psychotic symptoms in individuals with ASD (Nylander et al. 2008). de Bruin et al. failed to identify schizophrenia in their sample. However, it is persistently reported that patients with childhood-onset schizophrenia may display premorbid features of ASD and an association between childhoodonset schizophrenia and PDD NOS is postulated (Rapoport et al. 2009).

Neither anorexia nervosa nor bulimia was diagnosed in our sample. Eating problems are reported to be common in children with ASD (Schreck et al. 2004). However, children in our study did not exhibit the cognitive aspects of anorexia nervosa, nor did any have a substantial weight loss or episodes of overeating. Studies report autistic traits in patients with anorexia nervosa (Wentz et al. 2005), however, we are not aware of studies systematically evaluating symptoms of anorexia nervosa and bulimia in an ASD population. 


\section{General Discussion}

There is some consistency between our data on comorbid psychiatric disorders in an ASD child population and the findings in other reports using similar methodology: The overall prevalence of disorders is high, and ADHD and anxiety disorders are reported to be common. There are also inconsistencies. These may be explained by sample differences. However, given the phenotypical overlap between comorbid disorders and ASD, it is equally likely that rate differences stem from dissimilarity in interpreting and defining psychiatric symptoms. Clearly, the diagnostic validity of comorbid psychiatric disorders in ASD warrants further research (Lecavalier et al. 2009).

We found, in line with Sigonoff et al., few associations between comorbid psychiatric disorders and child characteristics. As proposed by Sigonoff et al., this could mean that the core ASD deficits represent the dominant factors overshadowing association with child characteristics. Nevertheless, questionnaire studies have reported symptoms of GAD and separation anxiety to be more prevalent in children with higher cognitive level (Sukhodolsky et al. 2008), depressive symptoms to increase with age (Brereton et al. 2006), and hyperactivity to be more common in younger children (Sinzig et al. 2009). These results, along with our findings that OCD was more prevalent in older children, and that ODD/CD was associated with PDD NOS, encourage continued research into the way developmental level influences the prevalence and expression of comorbid psychiatric disorders in children and adolescents with ASD.

The most prevalent diagnostic groups in our study, anxiety and ADHD, also constitute the most frequent in the general child population and in children with learning disabilities (Heiervang et al. 2007; Costello et al. 2003; Dekker and Koot 2003). Although, ODD/CD was relatively less frequent in our sample, behaviour problems were reported to be common. Hence, the differential distribution of psychiatric disorders in our study could be seen as similar to that in other child populations. This could indicate that etiological factors underlying the development of psychiatric disorders are equally distributed in ASD and non-ASD child populations, as indicated by genetic studies (Gadow et al. 2008). The higher prevalence in ASD, though, might reflect increased vulnerability to develop additional psychopathology due to impairment in communication and abstract thinking in children with ASD. On the contrary, psychiatric disorders are reported to be overrepresented in first-degree relatives of individuals with ASD, suggesting a genetic link between ASD and additional psychiatric disorders (Bolton et al. 1998). Clearly, more knowledge on psychiatric comorbidity could provide clues for further investigation of genetic vulnerability in ASD.
Strengths and Limitations

This study has several strengths. The sample included children of all cognitive levels and main ASD subgroups. A standardized interview was used for diagnostic purposes, and it is plausible that an interview, as opposed to a questionnaire, allows for a better distinction between core ASD features and symptoms of comorbid disorders. However, we are not aware of studies that have compared the utility of standardized interviews to that of questionnaires for assessing psychiatric comorbidity and identifying associations between psychiatric symptoms and disorders and child characteristics.

The sample was not referred for psychiatric assessment or treatment, avoiding artificial high rates due to referral bias. However, the sample was recruited from a special school population, and it is likely that children attending special schools have more psychiatric disorders than children in population based samples. Our sample included a predominance of children with autistic disorder and relatively few with PDD NOS compared the latest estimates on relative sizes of ASD subgroups (Fombonne 2009). Further, there were many children with low intellectual level. Thus, our sample cannot be seen as representative for the entire population of children with ASD, and the generalizability of our results is limited.

The use of Kiddie-SADS, a diagnostic interview validated for use in typically developing children with a mental age over 6 years, may be considered inappropriate. However, specialized instruments for ASD child populations are few and not well validated or used in clinical practice. It is therefore our belief that a common child psychiatric interview provides important data on comorbidity in ASD and child and adolescent populations and elucidates diagnostic dilemmas, and thus, is valuable for the development of more appropriates instruments.

The few identified associations between comorbid psychiatric disorders and child characteristics could be due to our relatively small sample. Further, there were few children in the subtypes of psychiatric disorders and the comparison of prevalence rates to that in other studies should therefore be done with caution.

\section{Conclusion}

This study used a standardized interview to assess the prevalence of current comorbid psychiatric disorders in a special school population of children and adolescents with ASD. We found high prevalence of comorbid disorders, in particular anxiety disorders and ADHD. Few associations between psychiatric disorders and child characteristic were revealed. Comorbid psychiatric disorders and ASD show phenotypical overlap and may be difficult to distinguish. 
Diagnostic challenges could contribute to inconsistency in reported prevalence rates. Further research on the diagnostic validity of comorbid psychiatric disorders in children with ASD is needed before clinical implications can be drawn more fully.

Acknowledgments We gratefully acknowledge the children and their parents for their participation. We also thank Geir Høivang who had a crucial role in initiating and supporting the accomplishment of the study.

Open Access This article is distributed under the terms of the Creative Commons Attribution Noncommercial License which permits any noncommercial use, distribution, and reproduction in any medium, provided the original author(s) and source are credited.

\section{References}

Allen, G., \& Courchesne, E. (2001). Attention function and dysfunction in autism. Frontiers in Bioscience, 6, D105-D119.

American Psychiatric Association. (2000). Diagnostic and statistical manual of mental disorders, text revisited (DSM-IV-TR). Washington, DC: American Psychiatric Association.

Biederman, J., Petty, C. R., Dolan, C., Hughes, S., Mick, E., Monuteaux, M. C., et al. (2008). The long-term longitudinal course of oppositional defiant disorder and conduct disorder in ADHD boys: Findings from a controlled 10-year prospective longitudinal follow-up study. Psychological Medicine, 38, 1027-1036.

Bolton, P. F., Pickles, A., Murphy, M., \& Rutter, M. (1998). Autism, affective and other psychiatric disorders: Patterns of familial aggregation. Psychological Medicine, 28, 385-395.

Bradley, E., \& Bolton, P. (2006). Episodic psychiatric disorders in teenagers with learning disabilities with and without autism. British Journal of Psychiatry, 189, 361-366.

Brereton, A. V., Tonge, B. J., \& Einfeld, S. L. (2006). Psychopathology in children and adolescents with autism compared to young people with intellectual disability. Journal of Autism and Developmental Disorders, 36, 863-870.

Canitano, R., \& Vivanti, G. (2007). Tics and Tourette syndrome in autism spectrum disorders. Autism, 11, 19-28.

Costello, E. J., Mustillo, S., Erkanli, A., Keeler, G., \& Angold, A. (2003). Prevalence and development of psychiatric disorders in childhood and adolescence. Archives of General Psychiatry, 60, 837-844.

de Bruin, E. I., Ferdinand, R. F., Meester, S., de Nijs, P. F., \& Verheij, F. (2007). High rates of psychiatric co-morbidity in PDD-NOS. Journal of Autism and Developmental Disorders, 37, 877-886.

Dekker, M. C., \& Koot, H. M. (2003). DSM-IV disorders in children with borderline to moderate intellectual disability. I: Prevalence and impact. Journal of the American Academy of Child and Adolescent Psychiatry, 42, 915-922.

Diamond, A. (2005). Attention-deficit disorder (attention-deficit/ hyperactivity disorder without hyperactivity): A neurobiologically and behaviorally distinct disorder from attention-deficit/ hyperactivity disorder (with hyperactivity). Development and Psychopathology, 17, 807-825.

Dossetor, D. R. (2007). 'All that glitters is not gold': Misdiagnosis of psychosis in pervasive developmental disorders-A case series. Clinical Child Psychology and Psychiatry, 12, 537-548.

Fombonne, E. (2009). Epidemiology of pervasive developmental disorders. Pediatric Research, 65, 591-598.
Gadow, K. D., Devincent, C., \& Schneider, J. (2008a). Predictors of psychiatric symptoms in children with an autism spectrum disorder. Journal of Autism and Developmental Disorders, 38, $1710-1720$.

Gadow, K. D., Roohi, J., DeVincent, C. J., \& Hatchwell, E. (2008b). Association of ADHD, tics, and anxiety with dopamine transporter (DAT1) genotype in autism spectrum disorder. Journal of Child Psychology and Psychiatry, 49, 1331-1338.

Gardner, M. F. (2000). Receptive one-word picture vocabulary test2000 edition. Noavto, CA: Academic Therapy Publications.

Geschwind, D. H. (2009). Advances in autism. Annual Review of Medicine, 60, 367-380.

Ghaziuddin, M., Ghaziuddin, N., \& Greden, J. (2002). Depression in persons with autism: Implications for research and clinical care. Journal of Autism and Developmental Disorders, 32, 299-306.

Heiervang, E., Stormark, K. M., Lundervold, A. J., Heimann, M., Goodman, R., Posserud, M. B., et al. (2007). Psychiatric disorders in Norwegian 8-10-year-olds: An epidemiological survey of prevalence, risk factors, and service use. Journal of the American Academy of Child and Adolescent Psychiatry, 46, 438-447.

Holtmann, M., Bolte, S., \& Poustka, F. (2007). Attention deficit hyperactivity disorder symptoms in pervasive developmental disorders: Association with autistic behavior domains and coexisting psychopathology. Psychopathology, 40, 172-177.

Kaufman, J., Birmaher, B., Brent, D., Rao, U., Flynn, C., Moreci, P., et al. (1997). Schedule for affective disorders and schizophrenia for school-age children-present and lifetime version (K-SADSPL): Initial reliability and validity data. Journal of the American Academy of Child and Adolescent Psychiatry, 36, 980-988.

Lecavalier, L., Gadow, K. D., DeVincent, C. J., \& Edwards, M. C. (2009). Validation of DSM-IV model of psychiatric syndromes in children with autism spectrum disorders. Journal of Autism and Developmental Disorders, 39, 278-289.

Lee, D. O., \& Ousley, O. Y. (2006). Attention-deficit hyperactivity disorder symptoms in a clinic sample of children and adolescents with pervasive developmental disorders. Journal of Child and Adolescent Psychopharmacology, 16, 737-746.

Leyfer, O. T., Folstein, S. E., Bacalman, S., Davis, N. O., Dinh, E., Morgan, J., et al. (2006). Comorbid psychiatric disorders in children with autism: Interview development and rates of disorders. Journal of Autism and Developmental Disorders, 36, 849-861.

Lord, C., Pickles, A., McLennan, J., Rutter, M., Bregman, J., Folstein, S., et al. (1997). Diagnosing autism: Analyses of data from the autism diagnostic interview. Journal of Autism and Developmental Disorders, 27, 501-517.

Muris, P., Steerneman, P., Merckelbach, H., Holdrinet, I., \& Meesters, C. (1998). Comorbid anxiety symptoms in children with pervasive developmental disorders. Journal of Anxiety Disorders, 12, 387-393.

Nylander, L., Lugnegård, T., \& Hallerbäck, M. U. (2008). Autism spectrum disorders and Schizophenia spectrum disorders in adults-Is there a connection? A literature review and some suggestions for future clinical research. Clinical Neuropsychiatry, 5(1), 43-54.

Rapoport, J., Chavez, A., Greenstein, D., Addington, A., \& Gogtay, N. (2009). Autism spectrum disorders and childhood-onset schizophrenia: Clinical and biological contributions to a relation revisited. Journal of the American Academy of Child and Adolescent Psychiatry, 48, 10-18.

Roid, G. M., \& Miller, L. J. (1997). Leiter international performance scale-revisited: Examiners manual. Wood Dale, IL: Stoelting Co.

Schreck, K. A., Williams, K., \& Smith, A. F. (2004). A comparison of eating behaviors between children with and without autism. Journal of Autism and Developmental Disorders, 34, 433-438. 
Simonoff, E., Pickles, A., Charman, T., Chandler, S., Loucas, T., \& Baird, G. (2008). Psychiatric disorders in children with autism spectrum disorders: Prevalence, comorbidity, and associated factors in a population-derived sample. Journal of the American Academy of Child and Adolescent Psychiatry, 47, 921-929.

Sinzig, J., Walter, D., \& Doepfner, M. (2009). Attention deficit/ hyperactivity disorder in children and adolescents with autism spectrum disorder: Symptom or syndrome? Journal of Attention Disorders, 13, 117-126.

Sparrow, S. S., Balla, D. A., \& Cicchetti, D. V. (1984). Survey form manual: Vineland adaptive behaviour scales. Circle Pines, MN: American Guidance Service.

Sukhodolsky, D. G., Scahill, L., Gadow, K. D., Arnold, L. E., Aman, M. G., McDougle, C. J., et al. (2008). Parent-rated anxiety symptoms in children with pervasive developmental disorders: Frequency and association with core autism symptoms and cognitive functioning. Journal of Abnormal Child Psychology, $36,117-128$.

Weisbrot, D. M., Gadow, K. D., DeVincent, C. J., \& Pomeroy, J. (2005). The presentation of anxiety in children with pervasive developmental disorders. Journal of Child and Adolescent Psychopharmacology, 15, 477-496.

Wentz, E., Lacey, J. H., Waller, G., Rastam, M., Turk, J., \& Gillberg, C. (2005). Childhood onset neuropsychiatric disorders in adult eating disorder patients. A pilot study. European Child and Adolescent Psychiatry, 14, 431-437.

Wozniak, J., Biederman, J., Faraone, S. V., Frazier, J., Kim, J., Millstein, R., et al. (1997). Mania in children with pervasive developmental disorder revisited. Journal of the American Academy of Child and Adolescent Psychiatry, 36, 1552-1559.

Yoshida, Y., \& Uchiyama, T. (2004). The clinical necessity for assessing attention deficit/hyperactivity disorder (AD/HD) symptoms in children with high-functioning pervasive developmental disorder (PDD). European Child and Adolescent Psychiatry, 13, 307-314.

Zandt, F., Prior, M., \& Kyrios, M. (2007). Repetitive behaviour in children with high functioning autism and obsessive compulsive disorder. Journal of Autism and Developmental Disorders, 37, 251-259. 